\title{
Measurements of stimulated-Raman-scattering-induced tilt in spectral-amplitude- coding optical code-division multiple-access systems
}

\begin{abstract}
We measure the stimulated Raman scattering (SRS)-induced tilt in spectral-amplitude-coding optical code-division multiple-access (SAC-OCDMA) systems as a function of system main parameters (transmission distance, power per chip, and number of users) via computer simulations. The results show that SRS-induced tilt significantly increases as transmission distance, power per chip, or number of users grows.
\end{abstract}

Keyword: Code division multiple access; Code division multiplexing; Optical fibre communication; Stimulated Raman scattering 